\title{
Abkürzungsverzeichnis
}

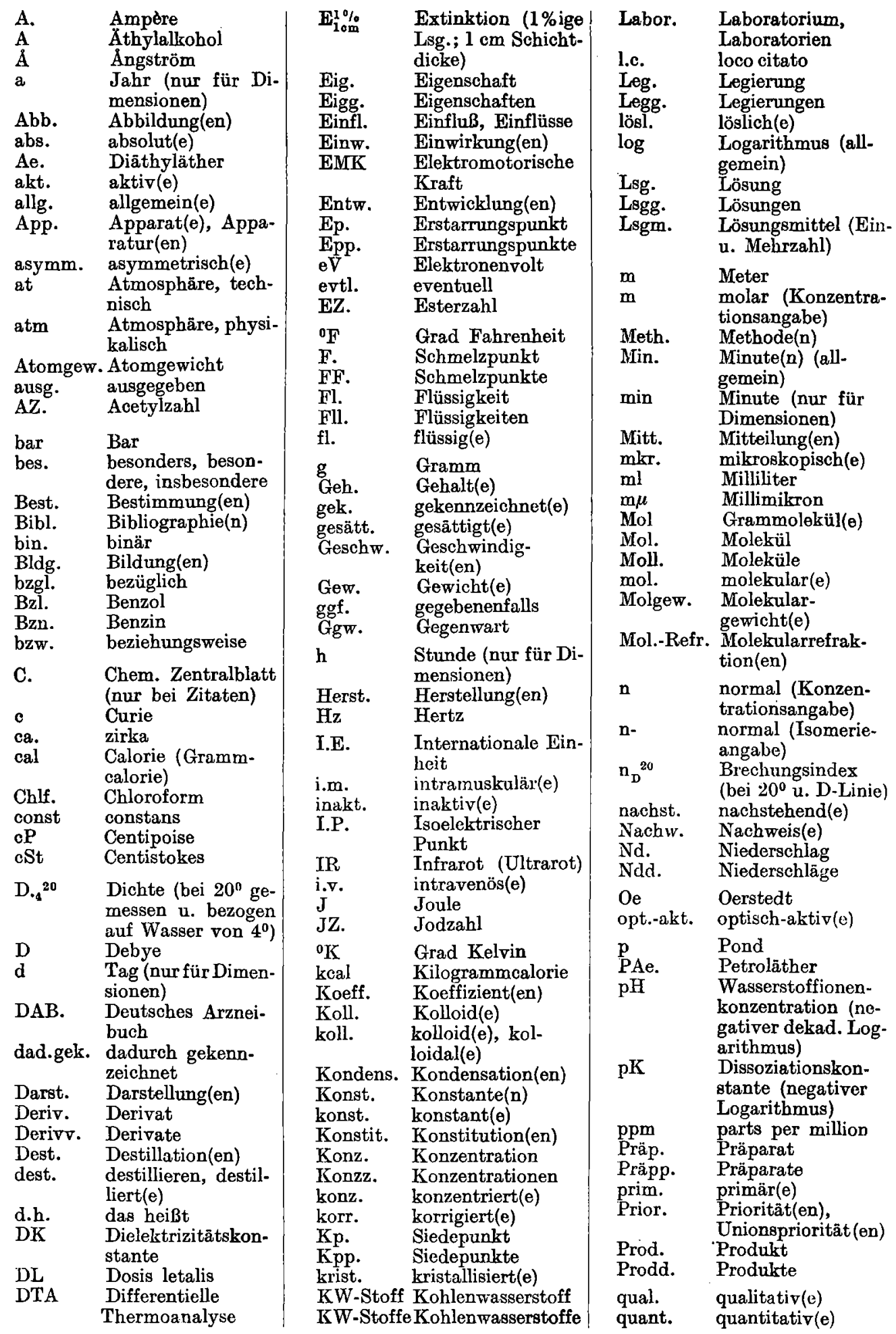




\begin{tabular}{lccc} 
Heft-Nr. & Seitenzahl & \multicolumn{2}{c}{ Ausgabedatum } \\
\hline 41 & $14525-14900$ & 11. Oktober & 1961 \\
42 & $14901-15268$ & 18. Oktober & 1961 \\
43 & $15269-15636$ & 25. Oktober & 1961 \\
44 & $15637-15988$ & 1. November & 1961
\end{tabular}

Herausgeber: Prof. Dr. Heinrich Berts ch, Berlin, u. Prof. Dr. Dr. h. c. Wilhelm Klemm, Manster. Chefredakteure und verantwortlich für den Inhalt: Dr. Willi Bar z (komm.), Berlin N 4, Schiffbauerdamm 19, Fernsprecher: 425571 , und Dr. Eugen Klever, Berlin W 30, Geisbergstraße 39, Fernsprecher: 249541 .

(c)

Verlag: Akademie-Verlag GmbH, Berlin W 8, Leipziger Str. 3-4 (Fernsprecher : 2204 41, Telex-Nr. 011773 Postscheckkonto Berlin 35021), und Verlag Chemie, GmbH, Weinheim/Bergstr. (Fernsprecher: Weinheim 3635 [Sammelnummer] ; Fernschreiber : 0465516 chemieverl wnh; Postscheckkonten: Frankfurt/M. 145314 u. Berlin [West] 7430). Bestellnummer: 1007/132. Das Chemische Zentralblatt erscheint wöchentlich. Preis des Jahrganges einschl. Autoren-, Patent-, Sach- u. Formelregister: DM 960, 一; einseitig bedruckte Ausgabe: DM $1050,-$. Abgabe der Zeitschrift nur mit sämtlichen Registern. Abbestellungen nur bis spätestens 6 Wochen vor Ablauf des Kalenderjahres.

Alle Rechte, auch die der Ubersetzung sowie der photomechanischen Wiedergabe, sind vorbehalten. All rights reserved (including those of translations into foreign languages). No part of this issue may be reproduced in any lorm, by photoprint, microfilm or any other means, without written permission from the publishers.

Die Wiedergabe von Gebrauchsnamen, Handelsnamen, Warenbezeichnungen usw. in diesen Heften berechtigt auch ohne besondere Kennzeichnung nicht zu der Annahme, daß solche Namen im sinne der Warenzeichen- und Markenschutz-Qesetzgebung als frel zu betrachten wären und daher von jedermann benutzt werden düriten.

Satz und 1)ruck: VEB Leipziger Druckhaus, Leipzig, III/18/203. 10/61.

Veróffentlicht unter der Lizenznummer ZLN 5002 des Ministeriums für Kultur. Hauptverwaltung Verlagswesen.

Printed in Germany.

Die Aufgabe von Anzeigen kann sowohl beim Akademie.Verlag Berlin, als auch beim Verlag Chemie GmbH, Weinheim/Bergstr. erfolgen 


\section{CHEMISGHES ZENTRALBLATT}

VOLLSTÄNDIGES REPERTORIUM

FƯR ALLE ZWEIGE DER REINEN UND ANGEWANDTEN CHEMIE

1830 gegründet

1897-1945 von der Deutschen Chemischen Gesellschaft fortgeführt

Im Auftrage der Deutschen Akademie der Wissenschaften zu Berlin, der Chemischen Gesellschaft in der DDR, der Akademie der Wissenschaften zu Göttingen und der Gesellschaft Deutseher Chemiker (in der BRD) herausgegeben von

Prof. Dr. Heinrich Bertsch und Prof. Dr. Dr.h.c. Wilhelm Klemm

\section{JAHRGANG}

\section{1}

SEITE 14525-15988

Chefredakfeure: Dr. Willi Barz (komm.) und Dr. Eugen Klover 